\title{
ON A GENERALIZATION OF THE RESOLVENT CONDITION IN THE KREISS MATRIX THEOREM
}

\author{
H. W. J. LENFERINK AND M. N. SPIJKER
}

\begin{abstract}
This paper deals with a condition on the resolvent of $s \times s$ matrices $A$. In one of the equivalent assertions of the Kreiss matrix theorem, the spectral norm of the resolvent of $A$ at $\zeta$ must satisfy an inequality for all $\zeta$ lying outside the unit disk in $\mathbb{C}$. We consider a generalization in which domains different from the unit disk and more general norms are allowed.

Under this generalized resolvent condition an upper bound is derived for the norms of the $n$th powers of $s \times s$ matrices $B$. Here, $B$ depends on $A$ via a relation $B=\varphi(A)$, where $\varphi$ is an arbitrary rational function. The upper bound grows linearly with $s \geq 1$ and is independent of $n \geq 1$. This generalizes an upper bound occurring in the Kreiss theorem where $B=A$.

Like the classical Kreiss theorem, the upper bound derived in this paper can be used in the stability analysis of numerical methods for solving differential equations.
\end{abstract}

\section{INTRODUCTION}

In the stability analysis of numerical methods for solving differential equations one is often faced with the question whether the norms of the powers of a given matrix are uniformly bounded. The Kreiss matrix theorem (see, e.g., $[3,6,9])$ provides an important tool for answering this question. One of the assertions of the theorem relates the inequality

$$
\left\|A^{n}\right\| \leq M_{0} \text { for } n=1,2,3, \ldots
$$

to the resolvent condition,

$$
\left\{\begin{array}{l}
(\zeta I-A) \text { is regular with } \\
\left\|(\zeta I-A)^{-1}\right\| \leq M_{1} \cdot d(\zeta, V)^{-1} \text { for all complex } \zeta \notin V .
\end{array}\right.
$$

Here, $A$ stands for a complex $s \times s$ matrix, $I$ is the $s \times s$ identity matrix, $\|\cdot\|=\|\cdot\|_{2}$ is the spectral norm (the matrix norm induced by the Euclidean norm on $\left.\mathbb{C}^{s}\right), V$ is the closed unit disk in the complex plane, and

$$
d(\zeta, V)=\min _{\xi \in V}|\zeta-\xi|
$$

Received November 6, 1989; revised June 21, 1990.

1980 Mathematics Subject Classification (1985 Revision). Primary 15A60, 65L20, 65M10; Secondary $30 \mathrm{~A} 10$.

The first author's research has been supported by the Netherlands organization for scientific research (NWO).

Offprint requests to: M. N. Spijker. 
By power series expansion it is easily seen that (1.1) implies (1.2) with $M_{1}=$ $M_{0}$. The Kreiss theorem asserts that, conversely, (1.2) implies (1.1) with $M_{0}$ depending on $M_{1}$ and $s$ only. Various authors (e.g., [10]) studied the size of (the optimal) $M_{0}$ as a function of $M_{1}, s$. Eventually it was proved by LeVeque and Trefethen [5] that (1.2) implies (1.1) with

$$
M_{0}=2 e s M_{1},
$$

and that no essential improvement upon this value is possible.

In this paper we shall generalize the result (1.3) in various directions. First of all, we shall be dealing with more general norms $\|\cdot\|$ than the spectral norm. Further, the set $V$ in (1.2) will be allowed to be a convex subset of the complex plane different from the unit disk. Finally, we shall derive an upper bound $M_{0}$ for the norms of the powers of $\varphi(A)$ instead of $A$ itself. Here $\varphi$ denotes an arbitrary rational function.

Like the classical Kreiss theorem, our generalization can be applied in the stability analysis of finite difference methods for solving partial differential equations. In the classical situation, $A$ is usually obtained by Fourier transformation and stands essentially for the so-called amplification matrix corresponding to the finite difference scheme under consideration. On the other hand, our generalization does not require such a transformation. It can be applied, more directly, with $\varphi(A)$ standing for the finite difference operator itself. In this fashion rigorous stability estimates can be derived, e.g., with respect to the maximum norm, in the numerical solution of initial-boundary value problems. For examples of such applications, see [4].

Theorem 2.2 in $\S 2$ contains the main result of this paper. It can be viewed as a generalization of (1.3). The proof of this theorem uses some ideas adopted from [5]. Further, it relies heavily on an upper bound for the arc length of the image $R(C)$ of a plane curve $C \subset \mathbb{C}$ under a rational function $R$.

In Lemma 3.1 of $\S 3$ we present the upper bound for $R(C)$ needed in $\S 2$. The proof of this lemma is based, among other things, on ideas taken from [9, pp. 397-402] and on Bézout's theorem (see, e.g., [11]). Further, $\S 3$ contains the technical Lemmas 3.2 and 3.3, which are also needed in the proof of Theorem 2.2 .

\section{A generalization OF THE RESUlt (1.3)}

In the following, $s$ is an arbitrary positive integer, and $\|\cdot\|$ denotes an arbitrary norm on the vectorspace $\mathbb{C}^{s, s}$ of all complex $s \times s$ matrices. We shall repeatedly use the following assumption on a plane curve $C$ in $\mathbb{C}$.

Assumption 2.1. There exist a positive integer $\nu$ and real coefficients $\alpha_{j, k}$ (for $j \geq 0, k \geq 0, j+k \leq \nu)$ with $\alpha_{j k} \neq 0$ for some $j, k$ satisfying $j+k=\nu$, such that $C$ is a subset of

$$
\{\zeta: \zeta=\xi+i \eta \text { with } \xi, \eta \in \mathbb{R} \text { and } K(\xi, \eta)=0\} .
$$


Here,

$$
K(\xi, \eta)=\sum_{0 \leq j+k \leq \nu} \alpha_{j k} \xi^{j} \eta^{k}
$$

This assumption will allow us to use Bézout's theorem, already mentioned in the introduction, in the proof of Lemma 3.1.

We now formulate our generalization of the assertion in $\S 1$ that (1.2) implies (1.1), (1.3).

Theorem 2.2. Assume

(2.1.a) $\varphi$ is a rational function which is not constant on $\mathbb{C}$, and $S$ is the set defined by

$$
S=\{\zeta: \zeta \in \mathbb{C} \text { and } \varphi \text { is regular at } \zeta \text { with }|\varphi(\zeta)| \leq 1\} \text {, }
$$

$V$ is a compact, convex subset of $S$,

(2.1.c) the boundary $C=\partial V$ of $V$ satisfies Assumption 2.1,

$$
\varphi^{\prime}(\zeta) \neq 0 \text { for all } \zeta \in(\partial V \cap \partial S),
$$

(2.1.e) $\quad A$ is a complex $s \times s$ matrix satisfying (1.2) for a given $M_{1}>0$.

Then

$$
\left\|\varphi(A)^{n}\right\| \leq \gamma M_{1} s \quad(n=1,2,3, \ldots),
$$

where $\gamma$ depends only on $\varphi$ and $V$ (and not on $A, s, n, M_{1}$, or $\left.\|\cdot\|\right)$.

Proof. 1. Assume (2.1) and let $n$ be a given positive integer.

By a version of the Hahn-Banach theorem (see, e.g., [8]), there exists a linear functional $F: \mathbb{C}^{s, s} \rightarrow \mathbb{C}$ with

$$
1=\|F\|:=\sup \left\{|F(B)|: B \in \mathbb{C}^{s, s} \text { with }\|B\| \leq 1\right\}
$$

and

$$
F\left(\varphi(A)^{n}\right)=\left\|\varphi(A)^{n}\right\| .
$$

In the following we assume $F$ is such a functional and consider the quantity $F\left(\varphi(A)^{n}\right)$.

In order to express $F\left(\varphi(A)^{n}\right)$ in terms of a Cauchy integral, we will define a suitable family of paths.

Let $\zeta_{1}, \zeta_{2}, \ldots, \zeta_{a}$ be those points on $\partial V$ for which $\varphi^{\prime}(\zeta)=0$.

2. First consider the case where there exists some $\zeta_{0} \in \operatorname{int}(V)$. Let, for $\alpha>0$ and $j=1,2, \ldots, a$,

$$
V^{j}=\left\{\zeta: \zeta \in \mathbb{C} \text { and }\left|\zeta_{0}+t\left(\zeta_{j}-\zeta_{0}\right)-\zeta\right| \leq \alpha \text { for some } t \in[0,1]\right\}
$$

and

$$
V_{0}=V \cup V^{1} \cup V^{2} \cup \cdots \cup V^{a}
$$


By assumptions (2.1.b,d) we can choose $\alpha$ such that the corresponding set $V_{0}$ is contained in $S$ and $\varphi^{\prime}(\zeta) \neq 0$ for all $\zeta \in \partial\left(V_{0}\right)$.

The assumptions of Lemma 3.2 are satisfied with $C=\partial\left(V_{0}\right)$ and $\zeta_{0}$ as above. Hence, $\partial\left(V_{0}\right)$ is equal to the range of a positively oriented Jordan curve $\Gamma_{0}$, parametrized by $\zeta=z_{0}(t), 0 \leq t \leq 1$. Here, $z_{0}$ satisfies (3.1) with some $m=m_{0} \geq 1, \mu_{1}=\mu_{1,0}>0, \mu_{2}=\mu_{2,0} \geq 0$, and $\tau_{j}$ satisfying $0=\tau_{0}<\tau_{1}<\cdots<\tau_{m}=1$.

We define our family of paths $\Gamma_{\varepsilon}$ by

$$
z_{\varepsilon}(t)=(1+\varepsilon)\left(z_{0}(t)-\zeta_{0}\right)+\zeta_{0} \text { for } \varepsilon>0 \text { and } t \in[0,1] .
$$

Set, for $\varepsilon>0$,

$$
W_{\varepsilon}=(1+\varepsilon)\left(V-\zeta_{0}\right)+\zeta_{0} \quad \text { and } \quad V_{\varepsilon}=(1+\varepsilon)\left(V_{0}-\zeta_{0}\right)+\zeta_{0} .
$$

Using the convexity of $V$ and the fact that $\zeta_{0} \in \operatorname{int}(V)$, we can see that

$$
V \subset \operatorname{int}\left(W_{\varepsilon}\right) \subset \operatorname{int}\left(V_{\varepsilon}\right) \text { for all } \varepsilon>0 .
$$

Further, using the fact that the interior of the Jordan curve $\Gamma_{0}$ is equal to $\operatorname{int}\left(V_{0}\right)$, we conclude that

(2.4.b) the interior of the Jordan curve $\Gamma_{\varepsilon}$ equals $\operatorname{int}\left(V_{\varepsilon}\right)$ for all $\varepsilon>0$.

Hence, for $\varepsilon$ sufficiently small, the curve $\Gamma_{\varepsilon}$ and its interior contain no poles of $\varphi$, whereas the spectrum of $A$ is contained in $V$ by (2.1.e). We may thus integrate along $\Gamma_{\varepsilon}$ to obtain by the Cauchy formula (see, e.g., [1, p. 568])

$$
F\left(\varphi(A)^{n}\right)=\frac{1}{2 \pi i} \int_{\Gamma_{\varepsilon}} \varphi(\zeta)^{n} F\left((\zeta I-A)^{-1}\right) d \zeta
$$

Choosing $\varepsilon$ sufficiently small, say $0<\varepsilon \leq \beta$, we have additionally

$$
\varphi^{\prime}(\zeta) \neq 0 \text { for all } \zeta \text { on } \Gamma_{\varepsilon} \text {. }
$$

By partial integration and (2.3) we thus obtain, for $0<\varepsilon \leq \beta$,

$$
\left\|\varphi(A)^{n}\right\|=-[2 \pi i(n+1)]^{-1} \int_{\Gamma_{\varepsilon}} \varphi(\zeta)^{n+1} R^{\prime}(\zeta) d \zeta .
$$

Here,

$$
R(\zeta)=F\left((\zeta I-A)^{-1}\right)\left[\varphi^{\prime}(\zeta)\right]^{-1} .
$$

Since $F$ is linear, we see that $F\left((\zeta I-A)^{-1}\right)$ is a rational function with numerator and denominator having degree not exceeding $s$. Consequently, $R(\zeta)$ is a rational function with numerator and denominator of degree at most $(s+M)$, where $M$ depends only on $\varphi$.

We define

$$
\begin{gathered}
r=\max \left\{\left|\zeta-\zeta_{0}\right|: \zeta \text { lies on } \Gamma_{0}\right\}, \\
L=\max \left\{\left|\varphi^{\prime}(\zeta)\right|: \zeta \text { lies on } \Gamma_{\lambda} \text { for some } \lambda \in[0, \beta]\right\} .
\end{gathered}
$$


In view of (2.1.a,b) we obtain from (2.5) the upper bound

$$
\left\|\varphi(A)^{n}\right\| \leq[2 \pi(n+1)]^{-1}(1+\varepsilon r L)^{n+1} \cdot \int_{\Gamma_{\varepsilon}}\left|R^{\prime}(\zeta)\right||d \zeta| .
$$

3. We will bound the above integral by using Lemma 3.1. Assumption 2.1 is fulfilled by $C=z_{0}[0,1]$, with some integer, say $\nu=\nu_{0} \geq 1$, because of (2.1.c) and by the construction of $z_{0}$. Since $z_{\varepsilon}(t)=(1+\varepsilon) z_{0}(t)+\varepsilon \zeta_{0}$, we see that Assumption 2.1 also holds with $C=z_{\varepsilon}[0,1]$ and with the same integer $\nu=\nu_{0}$. Thus, the conditions of Lemma 3.1 are satisfied with $z=z_{\varepsilon}, m=m_{0}$, $\mu_{1}=(1+\varepsilon) \mu_{1,0}, \mu_{2}=(1+\varepsilon) \mu_{2,0}, \nu=\nu_{0}, \Gamma=\Gamma_{\varepsilon}$, and $R(\zeta)$ as defined in (2.5.b). Lemma 3.1 and (2.6) thus lead to

$$
\left\|\varphi(A)^{n}\right\|=[2 \pi(n+1)]^{-1}(1+\varepsilon r L)^{n+1} \sigma_{0} \rho,
$$

where

$$
\begin{gathered}
\sigma_{0}=2 \mu_{2,0} \mu_{1,0}^{-1}+4\left(m_{0}-\nu_{0}\right)+16 \nu_{0}(s+M), \\
\rho=\max \left\{|R(\zeta)|: \zeta \text { lies on } \Gamma_{\varepsilon}\right\} .
\end{gathered}
$$

The quantity $|R(\zeta)|$ appearing in (2.7.c) can be bounded as follows. By (2.4) we have

$$
\begin{aligned}
\min \left\{d(\zeta, V): \zeta \text { lies on } \Gamma_{\varepsilon}\right\} & =\inf \left\{d(\zeta, V): \zeta \notin \operatorname{int}\left(V_{\varepsilon}\right)\right\} \\
& \geq \inf \left\{d(\zeta, V): \zeta \notin \operatorname{int}\left(W_{\varepsilon}\right)\right\} \\
& =\min \left\{d(\zeta, V): \zeta \in \partial\left(W_{\varepsilon}\right)\right\}
\end{aligned}
$$

From this inequality, (2.1.b), and an application of Lemma 3.3 with $V, \zeta_{0}$, and $\varepsilon$ as under consideration, we obtain

$$
d(\zeta, V) \geq \varepsilon \sigma_{1}>0 \text { for all } \zeta \text { lying on } \Gamma_{\varepsilon},
$$

where

$$
\sigma_{1}=\min \left\{\left|\xi-\zeta_{0}\right|: \xi \in \partial V\right\}
$$

Since $\|F\|=1$ and the matrix $A$ satisfies the resolvent condition (1.2), we have in view of $(2.5 . \mathrm{b})$ the inequality

$$
|R(\zeta)| \leq M_{1}\left(\varepsilon \sigma_{1}\right)^{-1} \sigma_{2}^{-1} \text { for } \zeta \text { lying on } \Gamma_{\varepsilon}, 0<\varepsilon \leq \beta,
$$

where

$$
\sigma_{2}=\min \left\{\left|\varphi^{\prime}(\xi)\right|: \xi \text { lies on } \Gamma_{\lambda} \text { for some } \lambda \in[0, \beta]\right\}
$$

We choose $\varepsilon=(n+1)^{-1} \beta$ and conclude from (2.7) and (2.8) that (2.2) holds with $\gamma$ depending only on $\varphi$ and $V$.

4. In the remaining case, when $\operatorname{int}(V)=\varnothing$, we take $\alpha>0$ such that

$$
V_{0}=V \cup\left\{\zeta: \zeta \in \mathbb{C} \text { and }\left|\zeta-\zeta_{j}\right| \leq \alpha \text { for some } j \text { with } 1 \leq j \leq a\right\}
$$

is contained in $S$. For $\varepsilon>0$, the set

$$
\left\{\zeta: \zeta \in \mathbb{C} \text { and } d\left(\zeta, V_{0}\right)=\varepsilon\right\}
$$


is the range of a piecewise smooth, positively oriented Jordan curve $\Gamma_{\varepsilon}$, parametrized by $\zeta=z_{\varepsilon}(t), 0 \leq t \leq 1$, with $\left|z_{\varepsilon}^{\prime}(t)\right|$ independent of $t$. We may then proceed as in the case when $\operatorname{int}(V) \neq \varnothing$ to arrive at (2.2) with $\gamma$ depending only on $\varphi$ and $V$.

\section{THREE LEMMAS}

The upper bound (3.2) of the subsequent lemma was used in the proof of Theorem 2.2. It can be viewed as a generalization of a bound derived by LeVeque and Trefethen [5] on the arc length of the image of a circle in the complex plane under a rational function.

Lemma 3.1. Let $z:[0,1] \rightarrow \mathbb{C}$ be a continuous mapping, one-to-one on $(0,1)$, and let

$$
0=\tau_{0}<\tau_{1}<\tau_{2}<\cdots<\tau_{m}=1 .
$$

Assume

(3.1.a) $\quad z$ is twice continuously differentiable at all $t \in(0,1)$ with $t \neq$

$$
\tau_{j}(j=1,2, \ldots, m-1) \text {, }
$$

(3.1.b) $\left|z^{\prime}(t)\right|=\mu_{1} \quad$ for all $t \in(0,1)$ with $t \neq \tau_{j}(j=1,2, \ldots, m-1)$,

$$
\int_{0}^{1}\left|z^{\prime \prime}(t)\right| d t \leq \mu_{2}
$$

Here, $\mu_{1}, \mu_{2}$ are constants with $\mu_{1}>0$.

Let $C=z[0,1]$ satisfy Assumption 2.1, and let $\nu$ be as required in this assumption. Denote by $\Gamma$ the oriented curve in $\mathbb{C}$ with parametrization $\zeta=$ $z(t), 0 \leq t \leq 1$.

Let $P(\zeta), Q(\zeta)$ be polynomials of degree not exceeding $N$, with $N \geq 1$ and $Q(\zeta) \neq 0$ for all $\zeta$ on $\Gamma$. Put

$$
R(\zeta)=P(\zeta) / Q(\zeta)
$$

Then

$$
\int_{\Gamma}\left|R^{\prime}(\zeta)\right||d \zeta| \leq\left(2 \mu_{2} \mu_{1}^{-1}+4(m-\nu)+16 \nu N\right) \cdot \rho
$$

where

$$
\rho=\max \{|R(\zeta)|: \zeta \text { lies on } \Gamma\} .
$$

Proof. 1. We start from the inequality

$$
\int_{\Gamma}\left|R^{\prime}(\zeta)\right||d \zeta| \leq \mu_{1} \cdot \sum_{j=1}^{m} \int_{\tau_{j-1}}^{\tau_{j}}\left(\left|\operatorname{Re}\left\{R^{\prime}(z(t))\right\}\right|+\left|\operatorname{Im}\left\{R^{\prime}(z(t))\right\}\right|\right) d t .
$$

In view of the absolute values occurring in the right-hand member of (3.3) it is useful to study first the number of sign changes of the functions $\operatorname{Re}\left\{R^{\prime}(z(t))\right\}$ and $\operatorname{Im}\left\{R^{\prime}(z(t))\right\}$. 
In the following we shall be using freely the notation

$$
\zeta=\xi+i \eta \quad \text { with } \zeta \in \mathbb{C}, \xi \in \mathbb{R}, \eta \in \mathbb{R} .
$$

We thus can write

$$
|Q(\zeta)|^{4} \operatorname{Re}\left\{R^{\prime}(\zeta)\right\}=G(\xi, \eta)
$$

where

$$
G(\xi, \eta)=\operatorname{Re}\left\{\left[P^{\prime}(\zeta) Q(\zeta)-P(\zeta) Q^{\prime}(\zeta)\right][\overline{[Q(\zeta)}]^{2}\right\}
$$

is a polynomial, with real coefficients, in the variables $\xi, \eta$. Note that the degree of this polynomial does not exceed $(4 N-1)$. It is clear that the sign of $\operatorname{Re}\left\{R^{\prime}(\zeta)\right\}$ for $\zeta=z(t), 0 \leq t \leq 1$, coincides with the sign of $G(\xi, \eta)$.

We define any $\sigma \in(0,1)$ to be an essential zero if

$$
G(\xi, \eta)=0 \text { for } \xi+i \eta=z(\sigma)
$$

and for all $\varepsilon>0$ there exists a $t_{\varepsilon} \in[0,1]$ with $\left|t_{\varepsilon}-\sigma\right|<\varepsilon$ such that

$$
G(\xi, \eta) \neq 0 \text { for } \xi+i \eta=z\left(t_{\varepsilon}\right) \text {. }
$$

We shall show, in part 2 of the proof, that

$$
\text { the number of essential zeros does not exceed }(4 N-1) \nu \text {. }
$$

2. For arbitrary polynomials $P(\xi, \eta)$ we introduce the notation

$$
C[P]=\{\zeta: \zeta=\xi+i \eta \text { with } \xi, \eta \in \mathbb{R} \text { and } P(\xi, \eta)=0\}
$$

Let $K(\xi, \eta)$ be as in Assumption 2.1 with $C=z[0,1]$. Then there exist polynomials $L(\xi, \eta), K_{0}(\xi, \eta)$ such that

$$
K(\xi, \eta) \equiv L(\xi, \eta) K_{0}(\xi, \eta),
$$

(3.5.b) $\quad K_{0}(\xi, \eta)$ and $G(\xi, \eta)$ have no common factor of degree $\geq 1$ (in the domain of polynomials in two variables over $\mathbb{C}$ ),

$$
C[L] \subset C[G]
$$

(cf., e.g., [11]).

Let $\sigma$ be an essential zero. In view of (3.5.a,c) the corresponding $t_{\varepsilon}$ are such that $z\left(t_{\varepsilon}\right) \in C\left[K_{0}\right]$. By letting $\varepsilon \rightarrow 0$ we see that

$$
z(\sigma) \in C\left[K_{0}\right] \cap C[G] .
$$

By virtue of (3.5.b), it follows from Bézout's theorem (cf., e.g., [11, Chapter III]) that the number of points $z(\sigma)$ lying in the above intersection does not exceed $(4 N-1) \nu$. Since $z$ is a one-to-one mapping on $(0,1)$, we arrive at (3.4).

3. Let $j$ be given with $1 \leq j \leq m$. Denote by $\sigma_{1}<\sigma_{2}<\cdots<\sigma_{a-1}$ the essential zeros within $\left(\tau_{j-1}, \tau_{j}\right)$, and put $\sigma_{0}=\tau_{j-1}, \sigma_{a}=\tau_{j}$.

Let $k$ be given with $1 \leq k \leq a$. One easily sees that either

$$
\operatorname{Re}\left\{R^{\prime}(z(t))\right\} \geq 0 \text { for all } t \in\left(\sigma_{k-1}, \sigma_{k}\right),
$$


or

$$
\operatorname{Re}\left\{R^{\prime}(z(t))\right\} \leq 0 \text { for all } t \in\left(\sigma_{k-1}, \sigma_{k}\right)
$$

Consequently,

$$
\int_{\tau_{j-1}}^{\tau_{j}}\left|\operatorname{Re}\left\{R^{\prime}(z(t))\right\}\right| d t=\sum_{k=1}^{a}\left|\int_{\sigma_{k-1}}^{\sigma_{k}} \operatorname{Re}\left\{R^{\prime}(z(t))\right\} d t\right| .
$$

Introducing

$$
\begin{array}{lll}
x(t)=\operatorname{Re}\{z(t)\}, & & y(t)=\operatorname{Im}\{z(t)\}, \\
f(t)=\operatorname{Re}\{R(z(t))\}, & & g(t)=\operatorname{Im}\{R(z(t))\},
\end{array}
$$

we can write the $k$ th term in the above sum as

$$
\left|\int_{\sigma_{k-1}}^{\sigma_{k}} \operatorname{Re}\left\{\frac{f^{\prime}(t)+i g^{\prime}(t)}{x^{\prime}(t)+i y^{\prime}(t)}\right\} d t\right|
$$

Since $\left|z^{\prime}(t)\right| \equiv \mu_{1}$, we see that this term is also equal to

$$
\left(\mu_{1}\right)^{-2}\left|\int_{\sigma_{k-1}}^{\sigma_{k}}\left\{\left(x^{\prime} f+y^{\prime} g\right)^{\prime}-\left(x^{\prime \prime} f+y^{\prime \prime} g\right)\right\}\right| \text {. }
$$

By (3.2.b), the last expression can be bounded by

$$
\left(\mu_{1}\right)^{-2}\left\{2 \mu_{1}+\int_{\sigma_{k-1}}^{\sigma_{k}}\left|z^{\prime \prime}(t)\right| d t\right\} \rho
$$

It thus follows that

$$
\int_{\tau_{j-1}}^{\tau_{j}}\left|\operatorname{Re}\left\{R^{\prime}(z(t))\right\}\right| d t \leq\left(\mu_{1}\right)^{-2}\left\{2 \mu_{1} a(j)+\int_{\tau_{j-1}}^{\tau_{j}}\left|z^{\prime \prime}(t)\right| d t\right\} \rho,
$$

where we have written $a=a(j)$ to indicate the dependence of $a$ on $j$.

4. From (3.4) it follows that

$$
\sum_{j=1}^{m}[a(j)-1] \leq(4 N-1) \nu .
$$

Combining this relation with (3.6), we conclude

$$
\sum_{j=1}^{m} \int_{\tau_{j-1}}^{\tau_{j}}\left|\operatorname{Re}\left\{R^{\prime}(z(t))\right\}\right| d t \leq\left(\mu_{1}\right)^{-2}\left\{2 \mu_{1}[(4 N-1) \nu+m]+\mu_{2}\right\} \rho .
$$

It can be seen, by a similar reasoning as above, that the last inequality remains valid if $\operatorname{Re}\left\{R^{\prime}(z(t))\right\}$ is replaced by $\operatorname{Im}\left\{R^{\prime}(z(t))\right\}$. A combination of these inequalities with (3.3) yields (3.2).

The subsequent Lemma 3.2 was used in part 2 of the proof of Theorem 2.2. Since the lemma can be proved by a straightforward application of ideas taken from [2], we confine ourselves to giving only a sketch of its proof. 
Lemma 3.2. Assume

$C \subset \mathbb{C}$ is a compact set,

C satisfies Assumption 2.1,

(3.7.c) there exists $\zeta_{0} \notin C$ such that each half-line

$$
\left\{\zeta: \zeta=\zeta_{0}+t \exp (i \alpha) \text { for some } t>0\right\},
$$

where $0 \leq \alpha<2 \pi$, intersects $C$ at precisely one point.

Then $C$ is the range of a positively oriented Jordan curve $\Gamma$, parametrized by $\zeta=z(t), 0 \leq t \leq 1$. Further, $z$ can be chosen such that (3.1) holds with some $m \geq 1, \mu_{1}>0, \mu_{2} \geq 0$, and $\tau_{j}$ satisfying $0=\tau_{0}<\tau_{1}<\tau_{2}<\cdots<\tau_{m}=1$.

Sketch of the proof. 1. Let $C$ and $\zeta_{0}$ be as in (3.7). By (3.7.a,c), $C$ is the image of a positively oriented Jordan curve $\Gamma$.

2. Let $\xi_{0} \in C$. Using the result of part 1, (3.7.b), and the theory in [2, §II.4], we can conclude that, in a neighborhood of $\xi_{0}$, the set $C$ is equal to the range of a curve $z(t)=x(t)+i y(t),-\varepsilon \leq t \leq \varepsilon$, where $\varepsilon>0$ and $x$ and $y$ are functions that are real analytic on $[-\varepsilon, 0]$ and $[0, \varepsilon]$.

3. By a transformation of the independent variable $t$ it can be proved that for all $\xi_{0} \in C$ there exist a $\delta>0$ and a function $z\left(\xi_{0}, t\right)$ such that

(3.8.a) in a neighborhood of $\xi_{0}$, the set $C$ is equal to the range of the simple curve $z\left(\xi_{0}, t\right), t \in[-\delta, \delta]$,

$$
\left|\frac{\partial}{\partial t} z\left(\xi_{0}, t\right)\right|=1 \quad \text { for all } t \text { with }|t| \in(0, \delta]
$$

$$
\int_{-\delta}^{\delta}\left|\frac{\partial^{2}}{\partial t^{2}} z\left(\xi_{0}, t\right)\right| d t \text { is finite (but possibly improper). }
$$

4. On the basis of the results of parts 1 and 3 , it is possible to construct a curve $\Gamma$ as in the statement of the lemma.

The following lemma was used in part 3 of the proof of Theorem 2.2. It can be proved by using basic properties of convex sets (cf., e.g., [7]).

Lemma 3.3. Let $V$ be a closed, convex subset of $\mathbb{C}$, and let $\zeta_{0} \in \operatorname{int}(V)$. Define

$$
\sigma=\min \left\{\left|\zeta-\zeta_{0}\right|: \zeta \in \partial V\right\}
$$

and, for $\varepsilon>0$,

$$
W_{\varepsilon}=(1+\varepsilon)\left(V-\zeta_{0}\right)+\zeta_{0}
$$

Then $d(\zeta, V) \geq \varepsilon \sigma$ for all $\zeta \in \partial\left(W_{\varepsilon}\right)$.

Added in proof. Relation (1.3) has recently been improved to the optimal value $M_{0}=e s M_{1}$, see M. N. Spijker, On a conjecture by LeVeque and Trefethen related to the Kreiss matrix theorem, BIT (to appear). 


\section{BIBLIOGRAPHY}

1. N. Dunford and J. Schwartz, Linear operators. I, Interscience, New York, London, 1958.

2. K. Kendig, Elementary algebraic geometry, Springer-Verlag, New York, Heidelberg, Berlin, 1977.

3. H.-O. Kreiss, Über die Stabilitätsdefinition für Differenzengleichungen die partielle Differentialgleichungen approximieren, BIT 2 (1962), 153-181.

4. H. W. J. Lenferink and M. N. Spijker, On the use of stability regions in the numerical analysis of initial value problems, Math. Comp. 57 (1991), 221-237.

5. R. J. LeVeque and L. N. Trefethen, On the resolvent condition in the Kreiss matrix theorem, BIT 24 (1984), 584-591.

6. R. D. Richtmyer and K. W. Morton, Difference methods for initial-value problems, 2nd ed., Wiley, New York, London, Sydney, 1967.

7. R. T. Rockafellar, Convex analysis, Princeton Univ. Press, Princeton, NJ, 1970.

8. W. Rudin, Functional analysis, McGraw-Hill, New York, 1973.

9. G. A. Sod, Numerical methods in fluid dynamics, Cambridge Univ. Press, Cambridge, 1985.

10. E. Tadmor, The equivalence of $L_{2}$-stability, the resolvent condition, and strict $H$-stability, Linear Algebra Appl. 41 (1981), 151-159.

11. R. J. Walker, Algebraic curves, Springer-Verlag, New York, Heidelberg, Berlin, 1978.

Department of Mathematics and Computer Science, University of Leiden, P. O. BoX 9512, 2300 RA Leiden, The Netherlands 